\title{
Protée
}

\section{La réception immédiate des Demi-civilisés de J.-C. Harvey. Éléments de sociosémiotique du discours critique}

Josias Semujanga

Volume 27, numéro 2, 1999

La réception

URI : https://id.erudit.org/iderudit/030556ar

DOI : https://doi.org/10.7202/030556ar

Aller au sommaire du numéro

Éditeur(s)

Département des arts et lettres - Université du Québec à Chicoutimi

ISSN

0300-3523 (imprimé)

1708-2307 (numérique)

Découvrir la revue

Citer cet article

Semujanga, J. (1999). La réception immédiate des Demi-civilisés de J.-C. Harvey. Éléments de sociosémiotique du discours critique. Protée, 27(2), 23-33.

https://doi.org/10.7202/030556ar
Résumé de l'article

Cet article propose, à partir de la réception immédiate des Demi-civilisés, la construction d'un algorithme narratif et discursif pour le discours critique. De nature sociosémiotique, celui-ci s'articule autour de deux isotopies fondamentales et solidaires. Liée aux figures à connotation littéraire (genres, motifs, thèmes, formes, etc.), l'isotopie littéraire a pour fonction de rattacher le discours critique à un lieu appelé « littérature ». Elle est également liée à l'isotopie référentialiste et idéologique qui articule le discours critique à un ancrage historique et géographique. Ces deux isotopies fonctionnent comme des indices qui opposent la critique littéraire à d'autres types de critique (historique, sociologique) et construisent en synchronie la notion de valeur esthétique des textes pour une société et une époque données. 


\title{
LA RÉCEPTIO N IM M ÉDIATE DES DEMI-CIVILISÉSDEJ.-C. HARVEY ÉLÉMENTS DE SO CIO SÉMIOTIQUE DU DISCOURS CRITIQUE ${ }^{1}$
}

JOSIAS SEMUJANGA

\begin{abstract}
Un texte ne peut dire quelque chose du monde qu'en inscrivant le fonctionnement du lieu qui l'a rendu possible, qu'en mettant en jeu dans son énonciation les problèmes que pose l'inscription sociale de sa propre énonciation.
\end{abstract}

Dominique Maingueneau, 1993

\section{INTRODUCTION}

Quand Les Demi-civilisés parait, en 1934, l'auteur est déjà connu du public, car «son roman, Marcel Faure, avait déjà attiré sur lui, en 1922, quelques semonces de la part de la critique et des autorités bien pensantes de l'époque» (Rousseau, 1978: 343). Aussitôt publié, le roman est mis à l'Index dans le diocèse de Québec par le cardinal Jean-Marie Rodrigue Villeneuve, dont le décret de condamnation se trouve dans La Semaine religieuse:

[...] le Roman Les Demi-civilisés, de Jean-Charles Harvey, tombe sous le canon 1399, 3e du Code du Droit canonique. Conséquemment, ce livre est prohibé par le droit commun de l'Église. Nous le déclarons tel et le condamnons aussi de Notre propre autorité archiépiscopale. Il est donc défendu, sous peine de faute grave, de le publier, de le lire, de le garder, de le vendre, de le traduire ou de le communiquer aux autres (Can. 1398, 1). (Rousseau, 1978: 347)

Et l'auteur est invité, comme Galilée, à condamner son ouvre: «Après la déclaration de son Éminence le cardinal Villeneuve, publiée hier, je consens à retirer du marché mon dernier roman Les Demi-civilisés, et je prie les libraires et les éditeurs de bien vouloir en tenir compte" (Rousseau, 1978: 347).

Même si le texte du pronunciamiento épiscopal ne précise pas les raisons de la condamnation du livre de Harvey, on peut tout de même constater que l'intrigue de ce roman constitue une mise en accusation du clergé, dont le rôle social est très important à cette époque. Certains passages évoquent en effet la connivence de l'Église avec l'élite économique anglaise dans l'appauvrissement du peuple et stigmatisent l'hypocrisie du clergé qui ne remplit pas de façon désintéressée sa mission. 
À la sortie du roman, la réaction de la presse a été mitigée. Beaucoup de journalistes n'osent pas contredire l'Église. Ils demeurent silencieux. C'est qu'ils ont beaucoup à perdre dans ce débat très politisé où la liberté d'expression n'existe pas. Et il faudra attendre la réédition du roman, en 1962, pour que la réception positive voie le jour. De renégat qu'il était durant les années trente et quarante, l'auteur devient le précurseur de la modernité culturelle au Québec au cours des années soixante. Certes son roman continue d'être considéré comme une fable mal construite, mais l'histoire de la littérature québécoise associera désormais Jean-Charles Harvey à la figure de la lumière, que l'on opposera volontiers à l'obscurantisme de l'Église.

Mais dans cette étude, je voudrais me limiter uniquement à la réception immédiate qui, d'ailleurs, s'étend de 1934 à 1939 et dont l'impact ne pourrait être analysé en détail dans les limites d'un article comme celui-ci. En faisant une étude préliminaire de cinq textes publiés entre avril et septembre 1934 dans deux quotidiens montréalais - Le Canada et L'Ordre -, on peut dégager des paramètres communs à tous les textes de réception critique des Demi-civilisés.

De fait, les cinq textes reproduisent la vision d'une culture littéraire basée sur un conflit entre la mesure et l'excès. Ce qui autorise à dire que le texte critique convoque comme paradigme culturel un sujet classique de la culture française, en tant que possible esthétique actualisable, qui pourrait éventuellement servir dans un contexte différent, en l'occurrence celui qui prévalait dans le Québec des années trente. Pour tous ces textes critiques, le paradigme classicisme est suggéré comme figure permettant d'évaluer Les Demicivilisés en fonction des catégories littéraires préexistantes. C'est ce modèle qu'évoque Henri Girard lorsqu'il dit qu' «un homme de chez nous avec mesure et pondération a osé écrire pour tous les yeux et tous les cerveaux" (Girard, 1934: 2). Ailleurs, le roman de Harvey a été critiqué pour son manque de sobriété dans sa «critique sociale juste (mais) intempestive» (Sillet, 1934: 1), pour son «style ample» (Brunet, 1934: 1) et surtout pour son excès dans la peinture de mours. En gros, la critique immédiate des Demi-civilisés s'avère beaucoup plus sévère à l'endroit de Harvey romancier qu'à celui de Harvey écrivain. Autant elle s'entend pour dire que l'écriture de Harvey est celle d'un bon littérateur, autant elle reproche à l'auteur des Demi-civilisés d'avoir écrit un mauvais roman, d'avoir lié son projet d'étude des mœurs à une intrigue inadéquate.

Mais le texte le plus représentatif de cet échantillon est sans conteste celui de Lucien Parizeau (1934). Autant sur le plan de son propos que sur celui de son organisation syntaxique, il illustre les enjeux de la réception immédiate des Demi-civilisés. En effet, quand le texte de Lucien Parizeau a été publié, le roman de Harvey venait d'être frappé par l'Index. Et, sans doute, influencés par ce jugement mais aussi un peu dépassés par la nouveauté du roman même, beaucoup de journalistes ont alors reçu l'œuvre très froidement, tout en convenant de la nouveauté de son propos et de la qualité de son écriture. Mais l'évêque de Montréal n'ayant pas suivi celui de Québec, les journalistes montréalais ont eu un peu plus de marge de manœuvre que ceux de Québec.

En comparant les cinq textes critiques, on remarque que celui de Lucien Parizeau circonscrit mutatis mutandis l'ensemble des enjeux de la communication littéraire dans le paysage littéraire du Québec des années trente, et notamment les rapports de l'écriture romanesque avec ses propres régulations auto-nomisantes et avec l'idéologie de la morale chrétienne.

Dans le présent article, il s'agira de voir comment le critique Parizeau, en tant que journaliste culturel accrédité, propose des Demi-civilisés une lecture qui, tout en niant la valeur immédiate de l'œuvre même, y voit la promesse d'une valeur d'échange sûre. En donnant son avis au sujet du texte romanesque, d'entrée de jeu posé comme problématique, il reconfigure les trois actants de la communication littéraire: l'éditeur proposant un texte comme littéraire, le lecteur éventuel et le sujet de la culture en tant qu'interprétant commun entre les deux premiers. 


\section{RÉCEPTION IMMÉDIATE ET SITUATION LITTÉRAIRE}

La situation littéraire désigne ici le parcours narratif d'une œuvre de création esthétique dans l'histoire littéraire d'un corpus institutionnel (littérature québécoise, française, africaine, etc.). Et dans ce schéma narratif, la critique immédiate constitue la phase de la compétence dans cette séquence narrative de la situation littéraire. Pour mieux circonscrire la fonction du discours de la critique immédiate dans la transformation d'un nouveau texte en œuvre intégrée à un corpus institutionnalisé, je me servirai d'outils méthodologiques inspirés du schéma narratif général. Cela reviendra à montrer que la fonction de la critique immédiate, c'est-à-dire la réception positive (ou non) d'un nouveau texte, est de l'ordre d'une acquisition (ou non) de compétence. En reconnaissant à ce nouveau texte une valeur ajoutée (dite littéraire) d'échange au-delà de sa valeur d'usage immédiate, la critique suggère (aux lecteurs) qu'il s'agit d'un texte littérairement valable. Et, de ce fait, la publication d'un texte nouveau se présente comme une manipulation, c'est-à-dire comme l'action d'un destinateur - l'éditeur - sur un éventuel sujet de quête: le texte lui-même. Et dans ce processus de l'énonciation littéraire, l'anti-sujet serait ce qui empêche le nouveau texte d'être reconnu comme littéraire: les valeurs reconnues institutionnellement.

Au début de l'anecdote du roman, avant qu'il ne devienne directeur de revue puis un peu «libertin», le personnage central des Demi-civilisés rêve de journalisme et écrit un essai qui est refusé par le directeur de journal vers lequel il l'achemine. En effet, pour le journal, publier cet essai reviendrait à donner beaucoup d'importance à un jeune prétentieux qui, en plus de savoir écrire avec correction et bon sens, pousse l'inconvenance jusqu'à nourrir des idées qui ne sont pas celles de tout le monde. De là à prétendre que Harvey avait d'abord voulu écrire un essai, il y a évidemment une grande marge. Et cet épisode de l'essai refusé constitue uniquement le point de départ, la justification de l'anecdote. En posant que la «fable» est d'autant plus médiocre qu'elle est venue après coup, Parizeau pointe en quelque sorte du doigt le manque de culture et le conservatisme des directeurs de journaux comme étant la cause éloignée des excès du livre de fiction. Est-ce à dire que le critique a entrevu, dans cette possibilité d'imputer au contexte un excès du texte, un moyen pour contourner la portée de la mise à l'Index même? Qu'est-ce que cette fiction critique vient faire dans le processus d'intégration ou non du roman à la littérature?

D'un côté, un éditeur publie un roman pour qu'il soit lu et, de l'autre, un censeur en interdit ex cathedra la lecture. Si personne ne réagit, le roman fait long feu. Il ne sera pas lu, du moins dans l'immédiat. C'est gênant pour la critique journalistique, d'autant que l'auteur est lui-même rédacteur en chef du quotidien Le Soleil. Par sa dénonciation, dès la première ligne de son article, de la fable du roman, le critique Parizeau accepte le point de vue de Mgr Villeneuve: celui de l'autorité ecclésiastique. Puis en se posant lui-même, contrairement au libertin journaliste du roman, comme «ayant des mœurs d'honnête homme», il en arrive à occuper une position contradictoire à celle des patrons également tombés dans l'excès en congédiant l'écrivain à la suite de la mise à l'Index de son livre. Ayant dès lors renvoyé dos à dos, comme contradictoires, la demi-civilisation dans la fable et la demi-civilisation dans le contexte social, le critique peut en retour espérer un peu de clémence pour l'écriture des choses du quotidien:

Maintenant qu'il [Harvey] s'est soumis à l'autorité
ecclésiastique, - ce qui n'a pas empêché ses patrons d'agir en
saligauds - il deurait reprendre sous une autre forme les bonnes
idées de son œeuvre et les répandre, avec l'autorisation du
cardinal-archevêque de Québec. Cet homme de haute culture (il
s'agit de Mgr Villeneuve) sait mieux que personne à quel point
nous sommes, à certains égards, des demi-civilisés.

(Parizeau, 1934: 1)

De ce fait, la publication des Demi-civilisés dans le paysage littéraire du Québec des années trente constitue une ouverture que l'on peut facilement considérer comme une manipulation: un éditeur 
publie comme littéraire un texte qui remet en cause les fondements de l'institution, à tel point que celle-ci en interdit la lecture. Parizeau admet d'entrée de jeu, au moins stratégiquement, la pertinence de l'interdiction. Et c'est sur cette base qu'il entame un dialogue avec celui qui en est l'auteur. C'est à lui qu'il répond publiquement. C'est ce que confirme largement la finale, citée plus haut, qui place explicitement l'autorité religieuse dans le rôle de judicateur. Partant, le destinateur-manipulateur du texte critique de Parizeau et du roman de Harvey est Mgr Villeneuve, comme représentant de l'institution littéraire. L'enjeu est de taille, en effet, car même si ce livre est par "certains côtés courageux et juste», c'est, tout de même, «un ouvrage manqué» (ibid.). Mais la mise à l'Index, pour justifiée qu'elle soit, hypothèque lourdement le droit de parole d'«un journaliste de talent» et d' "un bon littérateur».

Étant donné le sérieux de la condamnation, la première phrase de l'article - «La fable de ce roman est d'autant plus médiocre que M. Harvey avait conçu son livre sous forme d'essai» (ibid.) - inscrit le propos du critique dans l'orthodoxie de la condamnation du censeur religieux. Ensuite seulement, le critique se permet d'avancer un point de vue plus nuancé: c'est «à cause de la forme romanesque que M. Harvey lui a donné après coup [que] c'est un ouvrage manqué» (ibid.). Ce qui revient à pointer dans l'œuvre manquée une sorte d'anti-sujet: une fable romanesque plaquée "après coup». Le héros de la quête qui s'amorce, le "livre qui devait être un essai», n'est pas pour autant un sujet de quête constitué. Car il n'a pas encore acquis la compétence qui lui permettrait de tenter un tour d'essai dans l'institution. Momentanément, ce qui est en place, c'est tout juste une proposition pour concilier les points de vue contradictoires en présence: l'écrivain Harvey voulait simplement écrire un essai pour «prouver que [ses] compatriotes [sont] presque tous des sauvages" (ibid.). On retiendra évidemment que le début de l'article laisse en plan l'idée d'un écart entre une volonté et sa transposition artistique. Et pour rendre recevable par Mgr Villeneuve cette proposition, dès le début du second paragraphe, le critique dénonce, comme «antithèse fondamentale du livre» de Harvey, les deux héros de l'histoire qu'il raconte.

En prenant en compte que le second paragraphe pose le roman comme "plus maladroit que nocif", puis convient que le héros croit que «la liberté morale est le pivot de la civilisation", alors que "cela ne veut rien dire», on arrive à mieux saisir la stratégie du critique. Celle-ci consiste simplement à minimiser l'importance de la «fable» tout en prenant bien soin de noter au passage que sous sa médiocrité elle contient tout de même des indices de respectabilité. À titre d'exemple, l'héroïne a beau être décrite comme une courtisane, elle est tout de même dite monogame et, dans ce qu'elle dit, tout n'est pas à rejeter. Malgré ses bêtises de jeune animal sensuel qui lui font accepter aveuglément l'éventualité des infidélités de son amant, elle n'en affirme pas moins que ces infidélités ne le détourneraient pas d'elle puisque les accidents de l'existence n'affecteraient pas l'essence de son être. Une fois ces citations faites pour caractériser les héros de la fable, une distinction dans le paragraphe suivant entre la nature animale et la nature humaine permet de poser qu'au-delà de ses égarements, le jeune animal attribuait à son amant une essence différente des accidents de son existence. Dans cette foulée, il est, pour ainsi dire, acquis que le roman est effectivement plus «maladroit que nocif».

Dans la seconde partie de l'article, il peut dès lors y avoir retour sur les lubies du personnage central du roman, au sujet de la liberté morale et de la civilisation dont il avait été question au début de la phase d'acquisition de compétence. En mettant en parallèle les deux passages, on reconnaît plus facilement que le critique se situe désormais du côté de ce que Harvey voulait écrire. Selon lui, l'auteur viserait du côté de l'intériorité morale, c'est-à-dire le côté du contenu qui serait resté latent:

Max croit que "la liberté morale est le pivot de la civilisation" (entre nous, cela ne veut rien dire) et confond les «fortes passions" et les "grands vices", comme s'il n'y avait qu'une différence de degré entre la syphilis et la santé. ( $\left.2^{e} \S\right)$

Partant de ce principe que la «liberté morale» est la condition de 
toute civilisation ou culture, M. Harvey remarque l'horreur de nos gens pour la beauté du corps humain, transposé dans les arts plastiques ou libéraux. Cette phobie du nu [...] vient de ce que nous confondons le corps et la chair. [...] une ignorance crasse des conditions de la beauté. (5e $\S$ )

Notons aussi que le point de vue radical du personnage lui est dicté par une quête de la liberté. Et celle-ci lui fait remarquer qu'elle est «Liberté» pour les vicieux autant que pour les passionnés comme lui. On se contentera de souligner également que le critique adopte ici, au début du cinquième paragraphe, une attitude qui implique plutôt que Harvey, le bon littérateur, a tout simplement commis une bévue accidentelle en imaginant ses personnages. Car Harvey part $d u$ principe de liberté morale pour critiquer la phobie du nu en art et cela implique qu'en réalité, il voulait écrire un essai au sujet des «vertus sociales" comme «bonté, amour, charité». Et de fait, l'inverse ne serait pas vrai, puisque l'animalité n'a pas d'intériorité et que ses personnages sont un «homme sans caractère» et une «jeune sensuelle». Une semblable réduction de l'objet visé par Harvey passe par l'équation d'égalité qui est faite entre la civilisation et ladite culture. Cette équation permet de retrouver dans la culture la division reconnue dans la civilisation en termes de nature animale/nature humaine. En témoignent des phrases comme:

C'est en ce sens que M. Harvey a raison; rien ne témoigne plus profondément de la déformation collective de la majorité de nos gens que le sens tout trivial qu'ils attachent aux formes nues [...] Mais pour montrer que le nu artistique est chaste par sa force d'attraction spirituelle, comme la laideur excessive est pudique par sa force de répulsion, M. Harvey n'avait pas besoin de nous montrer des hommes et des femmes dans le débordement de l'iuresse ou de l'amour [...]

[Et] L'intention de M. Harvey était évidemment tout autre que celle qui se dégageait de son livre. (Parizeau, 1934: 1)

Par ailleurs, le critique réactualise, autour de la distinction «corps/chair» communément évoquée pour traiter de la question du nu dans l'art, les isotopies «fable/essai». Et l'art chrétien apparaît comme un lieu de «transsubstantiation» où le corps, loin de sombrer dans la "chair", devient un indice de spiritualité: «Le christianisme a mis de la volupté dans le nu antique, mais il a donné aux yeux aveugles de Minerve un regard vers l'infini qu'ils n'avaient pas". Or, l'absence de regard vers l'infini chez le personnage féminin marque la fable romanesque. Et la finale du roman fait simplement que le personnage féminin retrouve, en suivant son ombre qui lui est apparue en songe, celui qui lui tient lieu de figure de rédempteur!

L'argumentation en faveur de la proposition de départ du critique culmine dans le passage affirmant que les bons romanciers catholiques ont réussi à peindre les vices de l'homme sans pour autant mériter d'être condamnés. Cet argument n'est évidemment pas une preuve que Harvey voulait faire un essai catholique. Il assure simplement au sujet de quête qu'est le livre de pouvoir transcender symboliquement la distinction entre fable et essai. Cela est, discursivement parlant, une performance. Harvey n'a pas su donner, en effet, aux yeux de son personnage féminin un regard vers l'infini et il n'a pas su résoudre artistiquement les problèmes de transposition qui se posaient à lui. Il aurait mieux fait de se contenter d'exprimer ses «idées directement, dans la langue et le style ordinaire des essais». En clair, la distinction artificielle «fable/essai» s'avère surmontée, en l'occurrence, dans des romans comme ceux de Mauriac et de Bernanos, qui sont des livres positifs, alors que celui de Harvey est "un livre négatif». C'est dire que «la langue et le style ordinaire» auraient pu permettre au bon littérateur qu'est Harvey d'écrire un essai dont la valeur littéraire aurait été acceptable. La transformation est donc réussie. De fait, en lieu et place de la «fable médiocre» à condamner à cause des personnages, il y a désormais, en perspective, un essai écrit dans la langue et le style ordinaire. On pourrait insister ici sur le fait que le paradigme «roman catholique» évoqué par Parizeau semble placer en situation d'infériorité culturelle le roman canadienfrançais. Et il est également nécessaire de se rappeler ici que l'enjeu n'est pas de valider le roman de Harvey mais de tirer parti de romans français jugés 
positivement par l'institution canadienne-française pour avaliser la proposition de départ du critique. Ce que Parizeau vise en l'occurrence, c'est simplement de faire de la langue française le paradigme de l'institution littéraire canadienne-française.

On ne saura jamais si Mgr Villeneuve a été d'accord avec Lucien Parizeau pour reconnaître qu'il faut savoir gré au livre de Harvey d'avoir fait la preuve que «nous sommes, à certains égards, des demicivilisés». Et ce n'est pas là l'important. Par contre, il est impérieux de comprendre pourquoi les dernières phrases de l'article de Parizeau actualisent par anticipation la non-condamnation du livre que pourrait maintenant écrire Harvey. C'est que le critique tout simplement combat la censure littéraire.

Nous avons déjà vu que ces phrases concèdent à Mgr Villeneuve, homme qui a une "haute culture», le privilège de sanctionner le propos de Parizeau. Discursivement, la sanction revient à Mgr Villeneuve parce que c'est de l'écriture centrée sur l'au-delà - la haute culture - qu'origine la condamnation de l'écriture centrée sur le quotidien de Harvey. Et narrativement, la sanction dans cette critique consiste non pas à invalider la mise à l'Index, mais au contraire, et tout simplement, à entériner la pertinence de la compétence mise en œuvre, eu égard à la manipulation, en l'occurrence la mise à l'Index comme interdiction de lire le livre de Harvey.

Cette compétence n'a pas consisté à dénoncer une hérésie - Mgr Villeneuve l'avait déjà fait! - mais bien à proposer l'utilité de faire la différence entre «un bon littérateur» (qui a su reconnaître son erreur), et un «ouvrage manqué», un ouvrage «nuageux» et «excessif» parce que les personnages n'établissent pas de distinction entre l'animalité et l'humanité. Puisque, selon le critique, cette intériorité ne manquerait pas à un essai ordinaire exposant les idées de Harvey au sujet du nu dans l'art, il devient ici nécessaire de bien circonscrire la fonction des dernières phrases de son texte dans la dynamique de l'article. Ne pas accorder à Harvey l'autorisation de répandre, sous une autre forme, ces bonnes idées, cela reviendrait à affirmer que la valeur d'usage du livre
- sa fable qualifiée de médiocre, mais somme toute accessoire - est plus importante que sa valeur ajoutée - il est bien écrit - et c'est un essai qui avait été projeté. Ne pas accorder cette autorisation, ce serait donc faire preuve de demi-civilisation. Ce dont ont fait preuve "les saligauds» de patrons de Harvey en le congédiant! Par contre, autoriser cette possibilité, c'est assumer culturellement la juste mesure de la condamnation civilisatrice des Demi-civilisés. En liant syntagmatiquement autorisation à culture, Parizeau ne met pas Mgr Villeneuve en contradiction avec son interdiction antérieure, faite au nom de la civilisation. Il l'amène, au contraire, à donner sa pleine valeur culturelle à son propos de critique en permettant à un bon littérateur comme Harvey de faire mieux. Mgr Villeneuve sanctionne, en somme, l'utilité de la différence faite entre fable et essai pour motiver sa mise à l'Index.

Cela étant compris, on réalise que la sanction s'avère opératoire littérairement. Il est inutile d'interdire la lecture des ouvrages littéraires, même les romans manqués comme Les Demi-civilisés. Après avoir joué astucieusement sur l'idée que Harvey avait d'abord voulu écrire un essai, Parizeau finit en proposant une lecture littéraire du roman qui entérine la condamnation civilisatrice de Mgr Villeneuve luimême, tout en la motivant culturellement! Est bien secondaire, en la circonstance, le fait que le critique convienne qu'il s'agit d'un ouvrage manqué.

L'important, c'est que le critique soit parvenu, à partir de critères d'écriture, à l'inscrire comme témoignant, à cause de ses imperfections mêmes, de la pertinence de la mise en place d'un paradigme culturel qui conviendrait à la société canadienne-française. Et, on en conviendra, le critique y est parvenu en faisant du savoir-écrire-la-langue le premier indice de ce paradigme.

De ce fait, l'autonomisation de l'écriture littéraire a impliqué la mise en place d'un simulacre d'interaction verbale: Mgr Villeneuve y est le locuteur absent et le critique s'y fait "humblement» son interlocuteur. L'effet de sens que révèle en définitive ce simulacre tient à la mise en place de deux figures d'écriture posées comme contradictoires et non 
articulées, soit la mise à l'Index de Mgr Villeneuve et le livre de Harvey. À la fin, même si la contradiction n'est pas abolie, son articulation est désormais possible. C'est le titre même du livre de Harvey -Les Demi-civilisés - qui donne sens à l'interdiction de Mgr Villeneuve. L'astuce tient à ce que, de bout en bout du texte, l'absent cède sa place aux figures d'écriture: sa mise à l'Index donnée comme écriture à décoder littérairement dans la première partie est lue par les «demi-civilisés» dont voulait parler Harvey dans la seconde.

Au terme de cette analyse, on peut se demander si Parizeau aurait pu imaginer que ce seraient les historiens de la littérature - et non pas Harvey - qui reprendraient sous une autre forme Les Demi-civilisés. Il est certes possible de tirer cette question de l'importance que donne Parizeau à l'histoire occidentale de l'art dans la deuxième partie de son article. Par contre, avant de prétendre y répondre, il faudra avoir à l'esprit que, sur ce chapitre, le roman de Harvey semble avoir vu plus juste en affirmant que la civilisation passerait au Canada français par un retour de ses héros, libérés moralement, aux valeurs sûres de ses ancêtres. En effet, au-delà de la critique immédiate, les historiens de la littérature allaient, au tournant des années soixante, faire des Demi-civilisés un roman touffu et pas très bien écrit, mais tout de même capital dans l'entreprise de libération de la littérature canadienne-française - en train de devenir québécoise - de l'emprise de l'idéologie cléricaloreligieuse de la langue gardienne de la foi.

On se retrouve, en somme, devant une évidence à tout le moins amusante: les historiens de la littérature ont reconduit l'idée d'une fable médiocre, mais pourtant ils ont fait du livre de Harvey une date charnière dans leurs tentatives pour rendre compte esthétiquement de l'évolution de la littérature québécoise. Serait-ce à dire que la notion de l'histoire impliquerait au préalable la fétichisation de certaines fables romanesques? La fonction de la critique immédiate serait-elle de rendre possible la notion de littérature, en faisant des fables romanesques des signes culturellement autonomes?

\section{DISCOURS CRITIQUE} ET VALEUR LITTÉRAIRE DES TEXTES

L'analyse de l'article de Parizeau autorise à dire, jusqu'à un certain degré, que le discours critique n'est pas très différent des autres discours argumentatifs produits dans une quelconque langue naturelle. Dès lors, la contradiction qu'articule cet article de Parizeau doit être définie fonctionnellement. Si son efficacité immédiate tient à ce qu'il reconnaît une valeur ajoutée à l'écriture de Harvey, il devient alors possible de proposer que la critique immédiate ait pour fonction de valoriser socialement l'activité de lecture de textes qui rendent vraisemblable l'existence de formes d'expressions littéraires autonomes par rapport à leur contenu thématique.

De ce fait, l'énonciation critique construit une possibilité de signification de type littéraire dans un univers socioculturel où se trouvent les lecteurs des textes. Et en cela, elle convoque la praxis littéraire qui est à la fois diachronique, notamment dans le cas du texte de Parizeau, par exemple par ses références au thésaurus littéraire, c'est-à-dire l'isotopie littéraire constituée par sa construction du simulacre contextuel de lecture: le Québec des années trente. Le discours critique fait ainsi de la compétence littéraire un lieu où une instance d'énonciation convoque les structures sémionarratives, notamment celles qui sont liées au genre et à la langue d'écriture, en montrant comment leur statut de devoirêtre littéraire est assumé par l'instance scripturale qui le transforme en savoir-faire littéraire et fait du nouveau texte un procès réalisé. Et tout se passe dès lors comme si la subtilité de l'écriture consistait à imiter sans reproduire le même modèle.

De cette manière, deux types d'isotopies se mettent en place. D'une part l'isotopie littéraire liée au genre et vis-à-vis de laquelle le texte montre son inscription dans un lieu discursif - la littérature -; d'autre part l'isotopie référentielle en rapport avec le public des lecteurs qui juge le respect/non-respect des modèles littéraires canonisés. Cette double isotopie qui surdétermine le discours critique renseigne sur [...] la manière spécifique, propre à chaque société, d'interpréter et d'assumer tant l'univers collectif que l'univers individuel (c'est- 
à-dire d'expliciter, pour elle, ce qu'elle entend par culture et nature, par vie et mort). (Greimas et Courtés, 1979: 356)

Ces indices littéraires apparaissent alors comme des variations qui, par exemple, opposent le discours littéraire au discours historique ou sociologique. Et avec les connotations esthétiques qui les accompagnent, de tels indices se constituent sur le plan collectif en taxinomies sous-jacentes au discours critique, dans la mesure où ces éléments sont repérables tant sur le plan lexical - l'emploi de tel ou tel mot du registre littéraire - qu'à celui des organisations syntaxiques du discours. De plus, en créant une telle illusion de la réalité que la parole du critique ne fait qu'organiser et construire, le discours critique institue une connotation sociale symbolique de la critique comme instance assurant la communication entre le sujet de l'écriture à l'œuvre dans le nouveau texte et le sujet esthético-littéraire collectif qui lui est contemporain. Ce mélange complexe, mais analysable à la condition de s'en tenir aux deux isotopies déjà posées - le discours référentialiste idéologique associé au sociolecte littéraire sur le renouveau de l'écriture -, permet de classer tel ou tel texte comme discours critique ${ }^{2}$ et comme sociosémiotique en ce sens qu'il est surdéterminé par un langage de connotation sociale de type esthéticolittéraire et référentiel.

Argumentatif et polémique, le discours critique présuppose en outre l'existence d'un niveau lexicosémantique de type littéraire le structurant et liant son plan figuratif à son plan syntaxique de telle sorte que, même si parfois les séquences narratives du texte critique peuvent paraître spécifiques, compte tenu de leur caractère argumentatif, elles n'en sont pas moins des variations - des algorithmes narratifs généraux.

Ainsi, Lucien Parizeau tient les deux pôles de ce processus dans son texte critique, dans la mesure où il montre que J.-C. Harvey ne se contente pas de mettre en règle seulement les critères de filiation et d'écart par rapport à l'esthétique romanesque de son époque, mais souligne en plus que la manière dont il le fait est en rapport avec l'univers de son roman: Les Demi- civilisés. Dire d'un texte qu'il est critique présuppose ainsi une discussion préalable sur le concept de littérarité ou de valeur esthétique des textes, car le discours critique entretient des rapports étroits avec la littérarité comme interprétant commun entre les usagers du discours dit littéraire.

Schématiquement parlant, toute la polémique sur le concept de littérarité sous-jacente, par hypothèse, à tout discours sur la littérature trouve là ses avatars et ses limites. Car, comme l'a très bien montré Thomas Aron (1984), un même texte contient en lui de nombreuses lectures possibles. Et l'auteur affirme par là, "surtout, l'irréductibilité, en dernière instance, du texte à ces lectures [...], et pas non plus à leur somme». En effet, si les deux perspectives ${ }^{3}$ - textualistes et contextualistes - sont «les clés du texte, le texte, pour ainsi dire, les «réserve», les efface en même temps qu'il les inscrit, et s'ouvre par là même à d'autres clés, à d'autres lectures possibles» (p.61). Ces virtualités de lectures sont donc des possibles littéraires à actualiser chaque fois qu'une lecture est faite de tel ou tel texte ${ }^{4}$.

De ce fait, la littérarité serait la compétence/la virtualité qui se manifeste comme une instance orientée vers un savoirfaire rendant possible le faireécrire ou la performance. Ce savoir-faire littéraire se présente comme un acte en puissance susceptible d'être convoqué, à partir de ce que l'on pourrait appeler les règles du schéma de communication esthético-littéraire, par tel ou tel sujet ${ }^{5}$. Ainsi, la compétence littéraire se présenterait comme le schéma qui, d'un côté, présuppose de la part du sujet qui s'attribue le savoir-écrire littéraire, en l'occurrence l'écrivain, une connaissance implicite des règles de la communication littéraire. Ce qui revient à dire que le savoir-faire de l'écrivain présuppose la connaissance de la compétence littéraire comme schéma: le devoirêttre littéraire dont un usage particulier participe à la constitution.

De l'autre côté, et aussi paradoxal que cela puisse paraitre, cette connaissance du fait littéraire ne porte pas uniquement sur le savoir-faire. Elle porte plutôt sur le devoirêtre littéraire, c'est-à-dire un ensemble de contraintes faites de prescriptions - le comment écrire 
qui se manifeste par les références nombreuses au genre, aux modèles canonisés, etc. - et d'interdictions - ce qu'il ne faut pas écrire - et dont la maîtrise manifestée dans un texte particulier constitue le vrai savoir-faire du sujet de l'écriture littéraire. Cela présuppose également que les objets-textes comme procès sont structurellement marqués par leur appartenance à la littérarité comme code et comme indice structurant le nouveau texte, tant sur le plan discursif que sur le plan narratif. Ainsi, pour être littéraire, tout texte présuppose d'une part un programme virtuel sur la littérarité et, de l'autre, une compétence particulière qui le réalise et que l'instance critique a pour objet de construire et de révéler.

Parizeau n'arrivait manifestement pas à lire que «la liberté morale est la condition de la civilisation». Or, il s'avère que c'est là le sens que construisent en interaction les deux amants des Demi-civilisés. Et faute de le comprendre, dans un premier temps, il prétendit que «cela ne veut rien dire» et que les deux amants étaient qui un "jeune fat sans caractère», qui un "jeune animal sensuel et intelligent». Puis, dans un deuxième temps, il a fait de cette «liberté morale» le principe de départ de la réflexion du bon littérateur Harvey sur la question du nu dans l'art. Et, dans ce contexte culturel, il a affirmé la pertinence dudit principe. Et il l'a actualisé alors à sa façon en reprenant essentiellement, et peut-être sans trop s'en rendre compte, les éléments mêmes qu'il a d'abord jugés globalement insignifiants! Mais à une exception près, c'est-à-dire que cette fois-ci il s'est spontanément situé dans une perspective historique, de telle sorte que la volupté du nu antique est venue remplacer la passion amoureuse et le regard vers l'infini.

Manifestement, l'invitation à oublier la fable et à la remplacer par un essai permet au critique de rapprocher deux visions contradictoires, non pas tant du point de vue de l'écriture que de celui de la nature humaine. C'est la qualité de l'écriture que l'on retrouve dans le livre de Harvey qui lui permet de suggérer à Mgr Villeneuve qu'un nouveau contrat de lecture est souhaitable pour tous. Et il fait de cette qualité d'écriture une preuve de ce qu'au fond Harvey partagerait la même vision de la nature humaine que Mgr Villeneuve. Si on y regarde deux fois, on saisit seulement, en effet, que cette idée de nature humaine se réaliserait dans la transposition artistique: «la perfection physique de la statuaire athénienne, l'harmonie des corps qu'elle idéalise, renferment [...] un élément spirituel». Tant et si bien, que c'est en écrivant "la langue et le style ordinaire des essais» que Harvey pourrait reprendre «les bonnes idées de son œuvre» (Parizeau, 1934: 1). Ce qui est évacué en même temps que la fable, c'est, en somme, que la nature humaine puisse être un effet de fable! Ce n'est pas la fable qui humanise la nature animale, mais c'est le christianisme qui, en donnant "aux yeux aveugles de Minerve un regard vers l'infini", a permis de définir, dans le temps, bien sûr, les conditions indiscutées de la beauté intemporelle visée par toute création artistique.

En s'entendant sur la qualité de l'écriture, on évacue donc momentanément la nécessité de choisir entre deux visions de la nature humaine: une vision centrée sur l'idée que la spiritualité se manifeste dans l'art et une autre centrée sur l'idée que l'art est créateur de spiritualité. Une telle traduction du roman de Harvey constitue évidemment une trahison. Mais cette trahison est pour la bonne cause. Elle permet au critique d'amener Mgr Villeneuve à convenir que le critique littéraire serait désormais en mesure de prendre en main le flambeau de la culture écrite. Il le tient encore aujourd'hui.

\section{CONCLUSION}

Pour conclure cette étude sur la réception immédiate des Demi-civilisés, retenons trois éléments importants de la critique en tant que discours sur la valeur littéraire des textes.

Notons tout d'abord que le discours critique fonctionne comme un contrat de type fiduciaire sur la valeur littéraire (la littérarité) des textes, contrat établi entre le destinateur - l'instance critique - et le destinataire: la collectivité des lecteurs. De fait, par son faire persuasif (argumentatif), le contrat du sujet 
critique est à la fois énoncif et énonciatif. Énoncif parce que ce contrat est manifesté dans le discours du critique lui-même tel qu'il est énoncé (références aux différentes figures de la littérature ou de l'art en général). Et énonciatif parce que le critique se trouve à montrer à son énonciataire dans quelle mesure le texte nouvellement publié reste indéniablement un faire-valoir pour établir un type de communication littéraire, communication basée sur l'existence d'un horizon d'attente entre le critique et son lectorat.

Rappelons ensuite que la littérature n'échappe pas, par ce fait même, aux contingences temporelles et aux déterminations extérieures. Car, comme le souligne Joseph Melançon,

[...] il est bien reconnu aujourd'hui qu'il ne suffit pas d'écrire un texte de fiction pour entrer dans la littérature. [Car] si poèmes ou romans ne sont pas lus et reconnus, ils ne font pas partie de cet ensemble de textes dits littéraires, et leur auteur ne peut prétendre être classé parmi les écrivains. (1996: 9)

Ainsi, la réflexion sur la réception immédiate des Demi-civilisés montre que la notion même de valeur esthétique d'un texte est une invention discursive chaque fois remaniée selon les déterminations idéologiques et identitaires propres à chaque espace et à chaque époque. Car les enjeux de valorisation, tantôt esthétiques, tantôt moraux, tantôt nationaux, l'emportent bien souvent sur ce qui est valorisé. Et la valeur littéraire d'hier n'est pas nécessairement celle d'aujourd'hui ni, probablement, celle de demain ${ }^{6}$. Mais il faut tout de même avoir constamment à l'esprit que les textes littéraires contiennent aussi certaines conditions spécifiques de leurs significations, qui les distinguent d'autres types de discours comme la philosophie ou l'histoire:

C'est que, malgré le rôle indéniable de l'institution littéraire dans

la détermination de la valeur littéraire, une idée de ce qu'est la littérature subsiste avec suffisamment de cohérence chez les lecteurs et les écrivains pour que l'on puisse considérer que la littérature est un concept qui, par-delà les époques, permet de désigner un usage spécifique de la langue: raconter des fictions selon certaines lois génériques. (Semujanga, 1997: 23)
À ce titre, même si toute énonciation justifiant l'existence des formes esthétiques d'un texte est une construction discursive assumée par une instance autre que littéraire, celle-ci prend nécessairement la littérature comme lieu de son énonciation. Et si, dans le cas de la réception des Demi-civilisés, toute désignation littéraire provient d'une surdétermination idéologique de la morale religieuse en tant que valeur ajoutée aux textes, il reste que les notions de littérarité et de valeur littéraire des textes sous-tendent inévitablement le discours critique. De ce fait, on est amené à considérer le discours critique comme un discours dont la spécificité réside dans son évaluation de l'usage littéraire de la langue à partir des considérations esthétiques, morales ou idéologiques propres à un espace historiquement déterminé.

Soulignons enfin que le travail du critique consiste à rapprocher le nouveau texte d'autres discours qui lui sont contemporains, discours idéologiques, politiques, religieux par rapport auxquels il prend nécessairement position. Et là, ce qui décide, en définitive, du caractère de critique littéraire d'un texte quelconque, c'est l'inscription des figures à connotation littéraire et des figures à connotation référentielle dans un contexte socioculturel de réception. De ce fait, la littérarité semble être moins une valeur donnée de l'extérieur au nouveau texte ou une caractéristique immanente du texte qu'un interprétant commun permettant l'interaction littéraire. C'est de cette interaction entre les énoncés littéraires et les énoncés socio-historiques que se constitue la critique littéraire. Et c'est en cela qu'elle est une sociosémiotique.

\section{N O TES}

1. Il s'agit de la critique immédiate que l'on considère comme une compétence dans la séquence narrative d'une situation littéraire (Milot et Semujanga, 1993) et non de la critique savante ou relecture qui, elle, est une sanction.

2. Le discours critique est à cet égard semblable, sur le plan narratif, au roman du code dont parle André Belleau (1980): les deux marquent 
avec insistance la relation de l'isotopie littéraire et de l'isotopie référentielle articulant le littéraire au social et vice versa.

3. Sans viser l'exhaustivité, rappelons le débat sur la question de la littérarité. Il a été de nombreuses fois soutenu, et notamment par l'École formaliste, que le caractère littéraire d'un texte serait inscrit dans ses structures internes (Jakobson, 1973; Milot et Roy, 1991a).

Contestée actuellement, cette thèse structuraliste a nourri beaucoup de réflexions sur la poétique littéraire et, dans une large mesure, elle a fait progresser de façon significative les recherches dans le domaine. Elle a permis, en effet, à la poétique textuelle de tenir compte de l'importance de l'organisation interne de tout discours et de façon plus marquée pour le texte dit littéraire en plus de son contenu dénotatif explicite.

4. Nous pensons ici à la relecture comme phase de sanction dans le schéma narratif de la situation littéraire (Milot et Semujanga, 1993), dont la fonction consiste à relittérariser le texte ancien, en montrant qu'il est de nouveau réactivable par le biais de points de vue critiques nouveaux. Il s'agit évidemment de la critique savante et non de la critique immédiate, seule phase qui nous intéresse ici. Néanmoins, la comparaison sert ici à distinguer les différentes manifestations de la littérarité dans les deux phases du schéma narratif d'une situation littéraire.

5. On peut parler ici du discours critique comme d'une praxis énonciative de type littéraire. En effet, le sociolecte littéraire qui est constamment convoqué par l'énonciateur/sujet critique est, mutatis mutandis, l'impersonnel dont parle Denis Bertrand dans ses travaux sur la praxis énonciative (1993) et qui, d'après cet auteur, est à la base du processus énonciatif.

6. Sur cette question, voir également Roland Barthes qui se pose la question de savoir si la forme suffit pour définir la notion de littérature et déplace la notion de la forme vers celle de l'interprétation : «La littérature possède-t-elle une forme, sinon éternelle, du moins transhistorique? Pour répondre sérieusement à cette question un instrument essentiel nous manque: une histoire de l'idée de littérature" (1964: 265).

\section{RÉFÉREN CES BIBLIO G RAPHIQ UES}

ARON, T. [1984]: Littérature et Littérarité. Un essai de mise au point, Paris, Annales littéraires de l'Université de Besançon. BARTHES, R. [1964] : Essais critiques, Paris, Seuil. Belleau, A. [1980] : Le Romancier fictif, Montréal, P.U.Q. BERTRAND, D. [1991]: "Référent interne et littérarité ", dans L. Milot et F. Roy (dir.), La Littérarité, Québec, P.U.L., 163-178;

[1993]: "L'impersonnel de l'énonciation. Praxis énonciative: conversion, convocation, usage", Protée, vol.21, nº 1, 25-32.

BOURDiEU, P. [1987] : «Lecture, lecteurs, lettrés, littérature», dans Choses dites, Paris, Minuit, 132-143.

BRUNET, B. [1934] : "Quand Québec se dessale», L'Ordre, 25 avril, 1. FRANCCEUR, M. [1989]: "Sémiotique de la littérature et esthétique des signes", Études littéraires, vol.21, n³.

GagnON, M.-A. [1978]: Jean-Charles Harvey, précurseur de la Révolution tranquille, Ottawa, Beauchemin.

GIRARD, H. [1934]: "Un livre de combat», Le Canada, 27 avril, 2.

GONZALO-Francoli, Y. [1982] : «La double réception des Demi-civilisés,
1934 et 1962 ", dans R. Giguère (dir.), Réception de textes littéraires québécois, Cahiers d'études littéraires et culturelles, vol. 7, Sherbrooke, Université de Sherbrooke, 42-67.

GreimAS, A. J. [1976] : Sémiotique et sciences sociales, Paris, Seuil;

[1979]: Introduction à l'analyse du discours en sciences sociales, Paris, Hachette Université.

GreimAs, A. J. et J. COURTÉS [1979]: Sémiotique. Dictionnaire raisonné de la théorie du langage, Paris, Hachette Université.

GRIGNON, C.-H. (interviewé par B. Brunet) [1934]: « Portrait: Grignon fait de la terre", L'Ordre, 19 mai, 1-2.

GRIVEL, C. [1973] : Production de l'intérêt romanesque, Paris, Mouton.

HaRVEY, J.-C. [1934] : Les Demi-civilisés, Montréal, Les éditions du

Totem.

HJelmslev, L. [1969]: Prolégomènes à une théorie du langage, Paris,

Mouton.

JAKOBSON, R. [1963] : Essai de linguistique générale, Paris, Minuit;

[1973]: Questions de poétique, Paris, Seuil.

LANDOWSKI, E. [1989a]: La Société réfléchie. Essais de socio-sémiotique,

Paris, Seuil;

[1989b]: «Du référent, perdu et retrouvé: Réponse à Mario Jori",

Revue internationale de sémiotique juridique, vol. 2, nº 6, 301-312;

[1991]: «Pour une problématique socio-sémiotique de la littérature»,

dans L. Milot et F. Roy (dir.), La Littérarité, Québec, P.U.L., 95-119.

Maingueneau, D. [1993]: Le Contexte de l'œuvre, Paris, Dunot.

MELANÇON, J. [1996]: Discours de l'université sur la littérature québécoise,

Québec, Nuit blanche éditeur.

Milot, L. [1992]: «Le sens critique de la critique. Le cas de J.-C.

Harvey", dans A. Hayward et A. Whitfield (dir.), Critique et littérature québécoise, Montréal, Triptyque.

MiLoT, L. et F. RoY (dir.) [1991a] : La Littérarité, Québec, P.U.L.;

[1991b]: «On Textual Reference to Writing and Its Correlation with Literary History", Poetics Todays, vol. 12, n4 4, 713-728;

[1993]: Inscriptions des figures de l'écrit. Relectures de romans québécois des Habits rouges aux Filles de Caleb, Québec, Nuit blanche éditeur, «coll.

Cahiers du CRELIQ".

Milot, L. et J. Semujanga [1993]: «Pour une narrativisation de la situation littéraire", Protée, vol.21, n 1, 57-64.

PARIZEAU, L. [1934]: "Le roman de Jean-Charles Harvey", L'Ordre, $1{ }^{\text {er }}$ mai, 1.

RousseAU, G. [1969]: Jean-Charles Harvey et son œuvre romanesque, Montréal, Centre éducatif et culturel;

[1978] : "Les Demi-civilisés. Roman de Jean-Charles Harvey",

Dictionnaire des ceuvres littéraires du Québec, tome II, Montréal, Fides, 343-349.

RoY, F. [1991] : «Le récit comme agent informateur du sujet. Lecture de L'Étranger de Camus ", Protée, vol. 19, no 1, 45-50.

SEMUjANGA, J. [1997]: "Et Présence africaine inventa une littérature", Présence africaine 156, 17-34;

[1996] : "Rhétorique de la critique littéraire africaine », Tangence 51, 81-97;

[1993] : "Onomastique littéraire et compétences de lecture», Protée, vol.21, n², 77-84.

SilleT, L. [1934]: "Printemps canadien ", L'Ordre, 28 septembre, 1.

Todorov, T. [1971]: Poétique de la prose, Paris, Seuil. 